\title{
AN ALGORITHM FOR CHECKING PROPERTY P FOR KNOTS WITH COMPLEMENTS OF HEEGAARD GENUS 2
}

\author{
R. P. OSBORNE ${ }^{\prime}$
}

\begin{abstract}
One of the most fundamental questions about knots is: If we know the topological type of the complement of a knot. is the knot determined? In this paper we give an algorithm for deciding if certain knots called tunnel number one knots are determined by their complements. This algorithm turns out to be practical and efficient in that it can be used on knots with ten crossings without the aid of a computer and one can expect to be able to handle knots with. say, twenty crossings with the aid of a desk-top computer.
\end{abstract}

We give an algorithm for deciding if a knot with a complement of Heegaard genus two is determined by its complement. This algoithm is efficient enough to be applied to knots with 10 crossings using about one hour of hand calculation. Knots with complements of Heegaard genus 2 include torus knots, two bridge knots and many others such as $8_{5}, 8_{10}, 8_{15}, 8_{20}, 8_{21}, 9_{16}, 9_{22}, 9_{44}, 10_{132}, 10_{145}, 10_{156}, 10_{161}$ from the knot tables. Knots with Heegaard genus two complements are the "tunnel number one knots". As a consequence of the application of our algorithm the only knots with 8 or fewer crossings not now known to have property $\mathrm{P}$ are $8_{16}, 8_{17}$ and $8_{18} .8_{17}$ is the only knot with fewer than 9 crossings that is not invertible [Ka]. $8_{18}$ is a beautifully symmetric knot about which little seems to be known except that it has a nontrivial second elementary ideal [Fox] so it has a complement of Heegaard genus 3. Perko's knot $10_{161}-10_{162}$ is easily shown to have property $P$ by our algorithm.

For an arbitrary knot there is no known algorithm for deciding if it has a complement of Heegaard genus two. However, we can give a procedure that has worked for every knot known to have Heegaard genus two complement on which we have tried it (more than 30 knots).

1. The algorithm. Let $\left\langle a, b \mid R_{1}\right\rangle$ be a geometric presentation of our knot complement $S^{3}-K$. Let $m$ be a geometric presentation of the meridian of $K$ and $l$ a geometric presentation of the longitude of $K$. Consider the geometric presentations $\left\langle a, b \mid R_{1}, m l^{q}\right\rangle$. Our knot has property $\mathrm{P}$ if the group presented is never trivial when $q \neq 0$. To decide if the group presented is trivial we first ask if there is an automorphism $\alpha$ of $F(a, b)$ that reduces the length of our presentation. This question can be decided by looking for automorphisms of the form $a \rightarrow b^{\varepsilon} a$ or

Received by the editors April 30, 1982 and, in revised form, August 28, 1982.

1980 Mathematics Subject Classification. Primary 57M25; Secondary 20F05.

${ }^{1}$ The results of this paper were presented in outline at the A.M.S. meeting held November 6-7, 1981 in Austin, Texas. 
$a \rightarrow a b^{\varepsilon}$ and $b \rightarrow b$ or of the form $a \rightarrow a$ and $b \rightarrow a^{\varepsilon} b$ or $b \rightarrow b a^{\varepsilon}, \varepsilon= \pm 1$. If no automorphism of this form reduces the length of our presentation then no automorphism of $F(a, b)$ reduces the length of our presentation. If $\left\langle a, b \mid \bar{R}_{1}, \bar{m} \bar{l}^{q}\right\rangle$ has shortest presentation under automorphisms of $F(a, b)$, we check to see if one of the two relators is a subword of the other. If neither is a subword of the other then the group presented is not trivial and so the knot has property $P$.

At this point in theory, the algorithm becomes much more complicated because if one relator is a subword of the other, one may have to examine the actual Heegaard diagram of the surgered knot. One looks for a way to cancel common subwords and begin again with a shorter presentation. For the examples tried the usual presentation of the longitude has yielded a presentation in which neither relator is a subword of the other. For this reason the entire computation can be made to be algebraic and one never needs to look at Heegaard diagrams.

See $\S 3$ for an example of the application of this algorithm.

2. Definitions and enabling theorems. Each compact, orientable 3-manifold may be constructed by attaching 2-handles to a handlebody. A handlebody together with a set of instructions determining the (isotopy class of) curves along which the 2-handles are to be attached is called a Heegaard diagram of the manifold. In this paper we shall often not distinguish between 3-manifolds differing only by attaching or removing a 3-handle because these operations are canonical.

Let $H$ be a handlebody and let $\alpha_{1}, \alpha_{2}, \ldots, \alpha_{m}$ be disjoint simple closed curves on $\partial H$. If we choose a complete system of meridian disks $D_{1}, D_{2}, \ldots, D_{n}$ for $H$ then each curve $\alpha_{i}$ determines a word in the free semigroup on $x_{1}, x_{1}^{-1}, x_{2}, x_{2}^{-1}, \ldots, x_{n}, x_{n}^{-1}$ by traveling around the curve $\alpha_{i}$ and recording $x_{j}$ or $x_{j}^{-1}$ when $\alpha_{i}$ crosses $D_{j}$ in the positive or negative direction. (With a little isotopic adjustment, we may assume that $\alpha_{i} \cap D_{j}$ is finite.) The set of cyclic words $\left\{R_{1}, \ldots, R_{m}\right\}$ thus obtained is called a presentation of $\alpha_{1}, \ldots, \alpha_{m}$. Such a presentation is said to be geometric. If the 3-manifold $M$ is obtained by sewing 2-handles to the curves $\alpha_{1}, \alpha_{2}, \ldots, \alpha_{k}$ then $\left\langle x_{1}, \ldots, x_{n} \mid R_{1}, \ldots, R_{m}\right\rangle$ is called a geometric presentation of $M$. Caution: Two different 3-manifolds may have the same geometric presentation, e.g. the lens spaces $L_{5,1}$ and $L_{5,2}$ both have the same geometric presentation $\left\langle a \mid a^{5}\right\rangle$.

THEOREM 2.1. The usual over presentation of the fundamental group of the complement of a knot is a geometric presentation of the knot complement $S^{3}-N(k)$.

In fact, topologists have a very strong affinity for geometric presentations because the usual way of getting a presentation is to use some handle decomposition of the space in question.

THEOREM 2.2. If $\left\langle x_{1}, \ldots, x_{n} \mid R_{1}, \ldots, R_{k}\right\rangle$ is a geometric presentation of $M$ and $R_{1}=x_{1}^{-1} R_{1}^{\prime}$ where $R_{1}^{\prime}$ is a word in the alphabet $x_{2}, x_{2}^{-1}, \ldots, x_{n}, x_{n}^{-1}$; then the presentation $\left\langle x_{2}, \ldots, x_{n} \mid \bar{R}_{2}, \ldots, \bar{R}_{m}\right\rangle$ obtained by replacing each occurence of $x_{1}$ in the relators $R_{2}, \ldots, R_{m}$ by $R_{1}^{\prime}$ is a geometric presentation of $M$.

For a proof of Theorem 2.2 see [O \& SII, Theorem 3.3]. 
THEOREM 2.3 (ZIESCHANG). If $\left\langle x_{1}, \ldots, x_{n} \mid R_{1}, \ldots, R_{m}\right\rangle$ is a geometric presentation of $M$ and $\phi$ is an automorphism of the free group $F\left(x_{1}, \ldots, x_{n}\right)$ such that the total length of the relators $\phi\left(R_{1}\right), \phi\left(R_{2}\right), \ldots, \phi\left(R_{m}\right)$ is minimal among all automorphisms, then $\left\langle x_{1}, x_{2}, \ldots, x_{n} \mid \phi\left(R_{1}\right), \ldots, \phi\left(R_{m}\right)\right\rangle$ is a geometric presentation of $M$.

For a proof see [Zie].

Before going further with our theorems we need to mention the problem of free cancellations. A geometric presentation may well have free cancellations in it. For example, the curve in Figure 2 below has a presentation $a a^{-1} a$ but this free cancellation cannot just be pulled back as can the free cancellation in Figure 1.

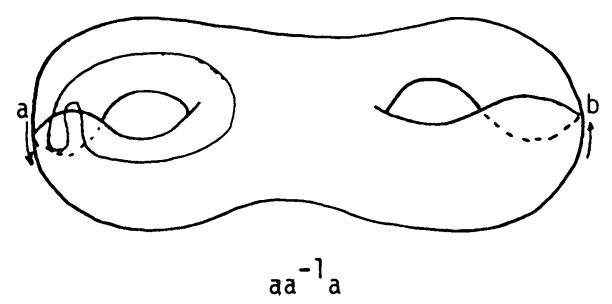

FIGURE 1

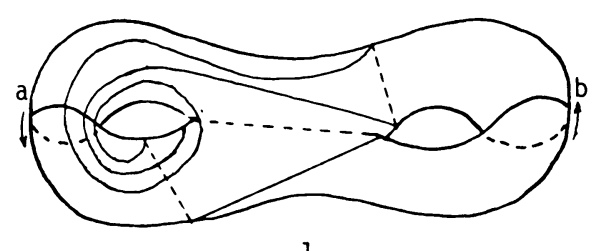

$\mathrm{aa}^{-1} \mathrm{a}$

FIGURE 2

This difficulty disappears when we use the shortest possible presentation because geometrically nontrivial free cancellations can be assumed not to appear in the shortest possible presentations under automorphism of the free group [O \& SII]. For this reason we simply ignore the free cancellation problem and treat all geometric relators as if free cancellations can always be done.

THEOREM 2.4. If $\left\langle x_{1}, \ldots, x_{n} \mid R_{1}, \ldots, R_{m}\right\rangle$ is a geometric presentation of $M$, $R_{1}, \ldots, R_{m}$ contains no free cancellations, and $x_{1} x_{2}$ is a subword of $R_{1}$, then the automorphism of $F\left(x_{1}, \ldots, x_{n}\right)$ defined by $x_{1} \rightarrow x_{1} x_{2}^{-1}, x_{i} \rightarrow x_{i}$ for $i \neq 1$ yields $a$ geometric presentation of $M$.

Proof. Since $x_{1} x_{2}$ is a subword of the relator $R_{1}$ there must be an arc $\lambda$ in the $P$-graph [O \& SI] of this presentation connecting $x_{1}^{+}$to $x_{2}^{-}$. Now a simple closed curve which is the boundary of a regular neighborhood of $x_{1}^{+} \cup x_{2}^{-} \cup \lambda$ will define the automorphism in question (see [O \& SII or Zie] for details on these simple closed curves). A free cancellation in the resulting presentation would appear only if some arc in the $P$-graph other than $\lambda$ intersected this curve more than once. This clearly does not happen.

Whitehead [Wh] has given an algorithm for deciding if some automorphism reduces the length of a presentation and for finding all such automorphisms (see also [M.K.S., p. 166]).

THEOREM 2.5 (HOMMA, OCHAI, TAKAHASHI). If $\left\langle a, b \mid R_{1}, R_{2}\right\rangle$ is a minimal length (under automorphisms), length $>2$, presentation of the 3-ball, then $R_{1}$ is a subword of $R_{2}$ or $R_{2}$ is a subword of $R_{1}$. 
This is Corollary 2 from [H.O.T.].

TheOREM 2.6 (ThURSTON). Surgery on a knot with Heegaard genus 2 complement yields no counterexample to the Poincaré conjecture.

As pointed out in [Thurs] this follows from the Smith conjecture and the work of Birman and Hilden.

3. An example. We illustrate the use of these theorems by applying them to show that the knot shown below is determined by its complement, so that by Theorem 2.6 it has property $\mathrm{P}$.

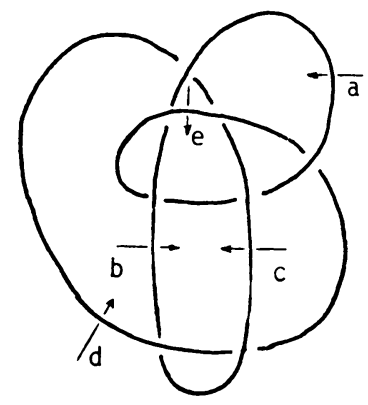

Figure 3

First we write down the over presentation,

$$
\left\langle a, b, c, d, e \mid c d b^{-1} d^{-1}, c^{-1} a e a^{-1} c d^{-1}, c^{-1} a c b^{-1} e^{-1} b, b e^{-1} a^{-1} e\right\rangle .
$$

The last relator is a defining relator for $b$, so we eliminate $b$ and substitute $e^{-1} a e$ for it.

$$
\left\langle a, c, d, e \mid c d e^{-1} a^{-1} e d^{-1}, c^{-1} a e a^{-1} c d^{-1}, c^{-1} a c e^{-1} a^{-1} e^{-1} a e\right\rangle .
$$

The second relator is a defining relator for $d$ so we eliminate $d$ and substitute $c^{-1} a e a^{-1} c$ for it.

$$
\left\langle a, c, e \mid a e a^{-1} c e^{-1} a^{-1} e c^{-1} a e^{-1} a^{-1} c, c^{-1} a c e^{-1} a^{-1} e^{-1} a e\right\rangle .
$$

Since there are no more defining relators we look for automorphisms that reduce the length of our presentation. The automorphism $a \rightarrow a, c \rightarrow c, e \rightarrow a^{-1} e$ reduces the length and gives

$$
\left\langle a, c, e \mid e a^{-1} c e^{-1} a^{-1} e c^{-1} a e^{-1} c, c^{-1} a c e^{-2} a e\right\rangle .
$$

The automorphism $a \rightarrow c a, b \rightarrow b, c \rightarrow c$ yields

$$
\left\langle a, c, e \mid e a^{-1} e^{-1} a^{-1} c^{-1} e a e^{-1} c, a c e^{-2} c a e\right\rangle .
$$

Next the automorphism $a \rightarrow e^{-1} a, c \rightarrow c, e \rightarrow e$ yields

$$
\left\langle a, c, e \mid e a^{-2} e c^{-1} a e^{-1} c, a c e^{-2} c e^{-1} a\right\rangle .
$$

Now do $a \rightarrow a, c \rightarrow c e, e \rightarrow e$ to get

$$
\left\langle a, c, e \mid e a^{-2} c^{-1} a e^{-1} c e, a c e^{-1} c a\right\rangle .
$$


Whitehead's algorithm tells us that there is no automorphism that shortens the length of this presentation. According to Theorem 2.3 this presentation is a geometric presentation of the complement of our knot. Note that the second relator is a defining relator for $e$. Eliminating $e=c a^{2} c$ we get

$$
\left\langle a, c \mid a^{2} c a^{-2} c^{-1} a c^{-1} a^{-2} c a^{2} c^{2}\right\rangle
$$

which again is a geometric presentation of our knot complement. We return to our original picture of the knot and find that a meridian is presented by $c$ and the longitude by $c^{-2} d e^{-1} c b^{-1} a^{-1} c a e c^{-1}$. If we follow these through out transformations we will get a geometric presentation of these curves in the final presentation. We get that the meridian is $c^{2} a^{2} c$ and the longitude is $c^{-1} a^{-2} c^{-2} a^{-2} c^{-1} a^{2} c a^{-1} c a c a c a^{-1} c a^{2}$. It follows that surgery on our knot that yields a homology 3-sphere has a geometric presentation

$$
\left\langle a, c \mid a^{2} c a^{-2} c^{-1} a c^{-1} a^{-2} c a^{2} c^{2}, \quad\left(c^{2} a^{2} c\right)\left(c^{-1} a^{-2} c^{-3} a^{-2} c^{-1} a^{2} c a^{-1} c a c a c a^{-1} c a^{2}\right)^{q}\right\rangle .
$$

It is now an easy matter to check that for $q \neq 0$ neither relator will be a subword of the other. It follows from Theorem 2.5 that nontrivial surgeries on our knot cannot yield $S^{3}$. By Theorem 2.6 then, no surgery on our knot can yield a simply connected 3-manifold.

4. Remarks. Using the above algorithm, Mark Willis [Will] has shown that the knots $8_{10}$ and $8_{15}$ from the table of knots in [Rolf] have property P. The author has checked $8_{21}, 10_{132}$ and $9_{16}$ for property P. Robert Huotari checked Perko's knot $10_{161}-10_{162}$ for a class project. Combining these results with those from [B \& M], [Sim] and [Tak] we now know that all knots with eight or fewer crossings have property $\mathrm{P}$ with the exceptions $8_{16}, 8_{17}$ and $8_{18}$. The knots $8_{17}$ and $8_{18}$ do not have complements of Heegaard genus 2 . In fact, these three knots are the only ones with fewer than 8 crossings to which our algorithm does not apply. The author is presently working on a computer program that will apply the algorithm to knots with less than 11 crossings to check those with Heegaard genus 2 complements for property P. It appears that the density of knots with Heegaard genus 2 complements decreases with increasing number of crossings. Noninvertible knots cannot have Heegaard genus 2 complements [Os].

\section{REFERENCES}

[B \& M] R. H. Bing and J. Martin, Cubes with knotted holes, Trans. Amer. Math. Soc. 155 (1971), $217-231$.

[Fox] R. H. Fox, A quick trip through knot theory, Topology of 3-Manifolds and Related Topics (M. K. Fort, Ed.), Prentice-Hall, Englewood Cliffs, N. J., 1962.

[H.O.T.] T. Homma, M. Ochiai and M. Takahasi, An algorithm for recognizing $S^{3}$ in 3-manifolds with Heegaard splittings of genus two, Osaka J. Math. 17 (1980), 625-648.

[Ka] A. Kawauchi, The invertibility problem for amphieheiral excellent knots, Proc. Japan Acad. 10 (1979), 399-402.

[M.K.S.] W. Magnus, A. Karrass and D. Solitar, Combinatorial group theory, Interscience, New York, 1966.

[Os] R. P. Osborne, Knots with Heegaard genus 2 complements are invertible, Proc. Amer. Math. Soc. 81 (1981), 501-502. 
[O \& SI] R. P. Osborne and R. S. Stevens, Group presentations corresponding to spines of 3-manifolds. I, Amer. J. Math. 96 (1974), 454-471.

[O \& SII] _ Group presentations corresponding to spines of 3-manifolds. II, Trans. Amer. Math. Soc. 234 (1977), 213-243.

[Rolf] D. Rolfsen, Knots and links, Publish or Perish, Berkeley, Calif., 1976.

[Sim] J. Simon, Some classes of knots with property P, Topology of Manifolds (Cantrell and Edwards, Eds.), Markham, Chicago, Ill., 1970.

[Tak] Moto-o-Takahashi, Two bridge knots have property P, Mem. Amer. Math. Soc. No. 239 (1981).

[Thurs] W. Thurston, The Smith conjecture, notes.

[Wh] J. H. C. Whitehead, On certain sets of elements in a free group, Proc. London Math. Soc. 41 (1936), 48-56.

[Will] Mark Willis, Masters Paper, Colorado State Univ., 1982.

[Zie] H. Zieschang, On simple systems of paths on complete pretzels, Trans. Amer. Math. Soc. 92 (1970), $127-137$.

Department of Mathematics, Colorado State University, Fort Collins, Colorado 80523 